\title{
Evolución del reporteo en sostenibilidad en Latinoamérica bajo los lineamientos del GRI (Global Reporting Initiative)
}

Evolution of sustainability reporting in Latin America under the guidelines of the Global Reporting Initiative (GRI)

\section{Evolução do Relatório de Sustentabilidade na América Latina sob as diretrizes do Relatório de Sustentabilidade GRI (Global Reporting Initiative)}

Juan Pablo Acevedo Téllew**

Universidad del Rosario, Colombia

Rafael Alejandro Piñeros ${ }^{* * *}$

Universidad del Rosario, Colombia

Cómo citar: Acevedo Téllez, J. A. y Piñeros, R. A. (2019). Evolución del reporteo en sostenibilidad en Latinoamérica bajo los lineamientos del GRI (Global Reporting Initiative). Signos. Investigación en Sistemas de Gestion, 11(2), 63-82. Doi: https://doi.org/10.15332/24631140.5082

* Artículo de investigación.

* Magíster en Dirección de Empresas, especialista en Gerencia de Empresas; licenciado en Electrónica. Bogotá, Colombia. Correo electrónico: Juan.acevedo@urosario.edu.co. Orcid: https://orcid.org/0000-0002-0714-7684

*** Magíster en Dirección de Empresas, administrador de empresas. Bogotá, Colombia. Correo electrónico: Rafael.pineros@urosario.edu.co. Orcid: https://orcid.org/0000-0002-3297-0752 


\section{RESUMEN}

Las guías para diseñar reportes de sostenibilidad establecidas por la GRI (Global Reporting Initiative) buscan generar compromiso en mitigar los impactos negativos de las compañías en las poblaciones, el medio ambiente, la economía, el desempeño social y los derechos humanos. Por ello, las preguntas centrales de esta investigación son: ¿̨Cómo ha evolucionado el número de reportes bajo el marco GRI en las principales economías de Latinoamérica desde 2010 hasta 2015? y ¿cómo se explica esta evolución? Para responder estas inquietudes se presenta un análisis descriptivo de la cantidad de reportes por año, país, sector y tamaño. Se hace una revisión de literatura, y se consulta la opinión de diferentes actores involucrados en la gestión de la responsabilidad social empresarial. Los hallazgos muestran una tendencia creciente en la actividad de reporteo y presentan las motivaciones detrás de estos esfuerzos.

Palabras clave: responsabilidad social empresarial, iniciativa de reporte global, Pacto Global, sostenibilidad.

\section{ABSTRACT}

The guidelines for the design of sustainability reports established by the Global Reporting Initiative (GRI) are intended to generate a commitment to mitigating the negative impacts of corporations on populations, the environment, the economy, social performance and human rights. Therefore, the core questions of this research are: How has the number of reports within the GRI framework has evolved in the different Latin American economies between 2010 and 2015? And how can this evolution be explained? To answer these questions, a descriptive analysis of the number of reports per year, country, sector and size is presented. A literature review is performed, and the opinion of different actors involved in the corporate social responsibility management is asked. The findings show a growing trend in the reporting activity and present the reasons behind these efforts.

Keywords: Corporate Social Responsibility, Global Reporting Initiative, Global Pact, Sustainability.

\section{RESUMO}

Os guias para projetar relatórios de sustentabilidade estabelecidos pela Global Reporting Initiative (GRI) procuram gerar um engajamento para mitigar os impactos negativos das empresas nas populações, no meio ambiente, na economia, no desempenho social e nos direitos humanos. Por isso, as questões essenciais desta pesquisa são: Como evoluiu o número de relatórios conforme o relatório GRI nas principais economias da América Latina a partir do ano 2010 até 2015? E como esta evolução pode ser explicada? Apresenta-se uma análise descritiva da quantidade de relatórios por ano/ país/setor/tamanho para responder essas dúvidas. É feita uma revisão da literatura e uma consulta à opinião dos diversos atores envolvidos na gestão da responsabilidade social empresarial. Os achados revelam uma tendência crescente na atividade de fazer relatórios e apresentam as motivações por trás desses esforços.

Palavras-chave: Responsabilidade social empresarial, Relatório de sustentabilidade, Pacto global, Sustentabilidade.

\section{INTRODUCCIÓN}

Organizaciones de diferentes tamaños y sectores se han embarcado en la tarea de elaborar reportes de 
sostenibilidad o RSE (responsabilidad social empresarial). Quiere esto decir que hay una intención creciente de ser socialmente responsables. De tal manera, inicialmente se define el concepto de responsabilidad social, se explican los motivos por los cuales una organización debería ser socialmente responsable y, finalmente, se plantea el objetivo de este estudio.

La RSE se define como la relación de la empresa con todos los grupos de interés y sus aportes en diferentes contextos para mitigar el impacto de sus operaciones no solo en la comunidad sino en todo su entorno. El objetivo es que las partes interesadas, involucradas en los contextos en los cuales la empresa realiza su operación, puedan convivir en armonía (Montoya y Martínez, 2012).

La RSE también ha sido comprendida como las prácticas que tienen las empresas para promover algún bien social más allá de los intereses de la organización, donde no solo se debe limitar al cumplimiento de la ley (Matten y Moon, 2008). Asimismo, su definición, sus criterios y su alcance son determinados desde la dirección (McWilliams y Siegel, 2001) pues básicamente cambian según las responsabilidades de la empresa y los países donde opera.

Desde la década de los noventa y con el auge de la globalización se hizo evidente que grandes multinacionales iniciaron maquilas principalmente en países en desarrollo con muy malas condiciones laborales. Frente a esto, la ONU emprendió la firma de tratados con el fin de generar conciencia social y responsabilizó a las compañías de los efectos generados en todo nivel entre esas poblaciones (Lozano, Raufflet, Barrera y García, 2012).

De la Cuesta (2004), fundamentado en la teoría de Carroll, encuentra que una empresa debería tener iniciativas en RSE por razones éticas, filantrópicas, económicas y legales. En cuanto a la ética las empresas deben dedicar sus esfuerzos no solo a los grandes accionistas, sino a todos los grupos de interés. Por cuestión de filantropía las empresas deben extender sus lazos a todas las partes interesadas, con el objeto de promover el bienestar social y mejorar la calidad de vida de estas. Por cuestiones económicas todas las empresas deben tener como fin generar riquezas para toda la comunidad, con lo cual contribuyen en mayor o menor medida al desarrollo sostenible de las naciones y el planeta. Por cuestiones legales los gobiernos deben estar involucrados en la RSE generando control para las empresas que, aunque deberían actuar voluntariamente en sus actividades de RSE, deben poder medirse y controlarse desde los diferentes estamentos del Gobierno nacional.

\section{Por qué reportar}

En sus primeros apartes el presente estudio ofrece una revisión de la literatura que abarca los principales motivos por los cuales las empresas toman la decisión de reportar sus actividades en responsabilidad social, describe las principales características de las guías de reporteo del Pacto Global y de la GRI (Global Reporting Initiative). Posteriormente presenta información cuantitativa que describe el comportamiento de las empresas que elaboran reportes en Latinoamérica. Después estos resultados se discuten a la luz de los hallazgos de la revisión bibliográfica y del análisis de las entrevistas de actores involucrados en RSE. Por último se presentan la discusión y las conclusiones que exponen los principales hallazgos y sus implicaciones en el mundo empresarial.

La presentación de informes de sostenibilidad es una actividad voluntaria que ha ganado una gran adhesión en el mundo corporativo (Thijssens, Bollen y Hassink, 2016). En este sentido, informar a los grupos de interés acerca del impacto de sus operaciones puede redundar en mejoras reputacionales y, de modo indirecto, en mejores resultados financieros. Es por esto que el reporteo de las actividades de sostenibilidad o responsabilidad 
social juega un papel cada vez más importante en el mundo corporativo.

Porter y Kramer (2006) mencionan que de la mano de iniciativas privadas, públicas y mixtas, a nivel nacional e internacional, la RSE se ha posicionado como una prioridad ineludible en la actividad empresarial. De tal manera, las iniciativas del Pacto Global y la GRI toman forma pues su objetivo es diseñar guías para que las empresas puedan informar los resultados de su gestión en términos económicos, sociales y ambientales a todas las partes interesadas. Así pues, su importancia radica en que es posible que la comunidad en general se informe acerca de las acciones que fomentan las empresas para que sus actividades sean responsables, amables y sostenibles con el medio ambiente. Consecuentemente, se busca que los informes sean una fuente de información verificable, medible y accesible para los grupos de interés.

En estudios anteriores acerca del tema, Gómez y Quintanilla (2012) mencionan tres razones para realizar estudios sobre el reporteo en responsabilidad social o la elaboración de informes de sostenibilidad empresarial: 1) la necesidad de sistemas de información y control sobre RSE; 2) el fortalecimiento de la confianza con los grupos de interés ya que estos sistemas deben ser evaluados, asegurados y auditados (Baker, 2005); 3) el cambio de la disciplina contable, que viene abordando criterios cualitativos, en los que se incluye información para los grupos de interés. Se estima que la RSE sería uno de los motores de este último cambio, y asimismo Archel y Husillos (2009) destacan la importancia de continuar con los estudios de caso que permitan un trabajo microanalítico y complementen los resultados de investigación sobre las tendencias de la información acerca de RSE.

Landrum y Ohsowski (2018) sostienen que las empresas comunican sus informes como parte de la gestión que hacen las partes interesadas para construir relaciones e influir en el comportamiento empresarial, mejorar la imagen de la empresa, buscar legitimidad, asumir la responsabilidad para señalar las actividades apropiadas y deseables, intentar un cambio de actitud y de comportamiento por parte de los consumidores con el fin de comunicar cómo la empresa, la comunidad y las partes interesadas dan sentido a su mundo y, por último, crear significado con las partes interesadas para construir la identidad de la empresa. También argumentan que el propósito general por el cual las empresas comunican las actividades en RSE es la búsqueda de un mayor rendimiento financiero.

Por otro lado, S. Dhaliwal, Zhen Li, Tsang y George Yang (2011) afirman que el reporteo es una forma en que las empresas pueden recuperar parte de su prestigio después de algún escándalo de corrupción o daño ambiental. También explican que la reputación de las empresas y sus ventas pueden verse afectadas a largo plazo si no llevan a cabo acciones de RSE.

English y Schooley (2014) mencionan que las empresas están cada vez más motivadas para abordar temas de sostenibilidad y riesgos relacionados debido a una mayor conciencia, el aumento de las regulaciones gubernamentales, las presiones de los grupos de interés y la buena voluntad que generan este tipo de actividades. También explican el tránsito que las empresas están asumiendo, de reportar voluntariamente a reportar obligatoriamente, no solo por las exigencias de los entes legislativos, sino por las de clientes e inversionistas.

Los informes de sostenibilidad son una oportunidad para que las empresas se dirijan a las audiencias en general, y si esto se integra en el gobierno corporativo, se tendrían cubiertas las relaciones entre empresa, accionistas y sociedad (Carroll, 1999; Correa, Flynn y Amit, 2004; Kolk, 2003). Se aclara también que es complicado ofrecer información que colme las expectativas de todas 
las solicitudes de información de los interesados. Sin embargo, dichos informes tendrán mayor validez si son verificados por una firma auditora externa, de acuerdo con lo mencionado en el estudio de Zorio, García-Benau y Sierra (2015).

Kolk (2008) explica que la elaboración de informes de RSE constituye un esfuerzo por reducir la tensión en las obligaciones de la empresa con los accionistas y los demás grupos de interés. En este sentido, la actividad del reporteo es ética y a la vez busca la generación de valor para todos los grupos de interés (Donaldson y Preston, 1995).

Kolk (2003) también señala, con base en los informes de sostenibilidad de Fortune Global 250, que los gobiernos corporativos de las empresas están cada vez más comprometidos con la transparencia empresarial y ofrecen información relevante para la formulación de estos reportes. Aclara, sin embargo, que esta información se presenta a nivel general, así que no determina las responsabilidades reales sobre las estructuras, y requiere la verificación externa de lo informado.

KLD Research y Analytics Inc., que monitorean el desempeño de las grandes empresas en RSE, indican que en los últimos veinte años el reporteo se ha incrementado de forma continua, principalmente en América del Norte y Europa respecto al resto del mundo (Moon, 2004; Ortas y Moneva, 2011; S. Dhaliwal et al., 2011), y aún más si es una empresa con alcance multinacional (Chapple y Moon, 2005). En los reportes de sostenibilidad la empresa privada, las ONG, los medios de comunicación y las entidades del Gobierno se están interesando por conocer, además de lo estrictamente legal, cualquier otro dato que las empresas quieran aportar de manera voluntaria (Chapple y Moon, 2005; McWilliams y Siegel, 2001). Es así como el reporteo de RSE toma relevancia en el entorno empresarial.

\section{Descripción Pacto Global y GRI}

Las iniciativas como el Pacto Global y la GRI surgen con el propósito de ayudar a empresas y Gobiernos a entender y comunicar sus contribuciones para el reto de la sostenibilidad. Los problemas de la sostenibilidad son de diferente índole: el cambio climático, los derechos humanos, la gobernanza y las iniciativas sociales, entre otros. Los informes constituyen una forma de recopilar esta información y de divulgarla para que sea de conocimiento público (Khan, Serafeim y Yoon, 2016). Su importancia radica en que es posible que la comunidad se entere acerca de cuánto están haciendo para que el planeta sea sostenible (GRI, 2017a).

El Pacto Global fue socializado al mundo en septiembre de 2000 por parte de la ONU, mediante el cual se invitóa las empresas a que voluntariamente acogieran y desarrollaran diez principios de las prácticas corporativas, como apoyo complementario para las iniciativas de políticas públicas (García, 2009). Surgió como una iniciativa que integra cuatro aspectos importantes: derechos humanos, derechos laborales, medio ambiente y lucha contra la corrupción.

Por otro lado, el GRI, a través de sus principios, funciona como una herramienta que permite establecer el desarrollo de las memorias que, junto con los contenidos básicos, permiten la construcción de los indicadores sobre los cuales se reportará la gestión realizada (Brown, De Jong y Levy, 2009). Dichos principios se dividen en dos grupos: el primero está constituido por los que determinan el contenido de la memoria (participación de los grupos de interés, contexto de sostenibilidad, materialidad y exhaustividad) y el segundo, por los que determinan la calidad de la memoria (equilibrio, comparabilidad, precisión, puntualidad, claridad y fiabilidad) (GRI, 2015).

La materialidad es el proceso de determinación de los asuntos que se consideran más importantes para la 
empresa y para los grupos de interés. Se sugiere que dicho proceso sea divulgado (Fasan y Mio, 2017). El GRI sugiere cuatro pasos para la definición de los temas más relevantes: 1) identificación de los aspectos y asuntos más relevantes; 2) priorización de los aspectos y asuntos más relevantes encontrados en el paso anterior; 3 ) validación, en la que se aplican los principios de exhaustividad y participación de los grupos de interés; 4) tras la publicación del documento, revisión de este por parte de la empresa pensando en el siguiente informe (Duque, Cardona y Rendón, 2013; RobecoSAM y GRI, 2015).

Landrum y Ohsowski (2018) sostienen que el de la GRI es el formato más utilizado a nivel mundial para elaborar informes de sostenibilidad. La guía estandariza la forma en que las empresas informan sobre indicadores económicos y de cumplimiento ambiental relacionados con prácticas laborales, derechos humanos, sociedad y responsabilidad del producto.

Como se ha anotado, son diversas las motivaciones que explican la intención de las compañías de incorporar prácticas de responsabilidad social, así como de elaborar informes de la misma naturaleza. Por tal motivo, es necesario estudiar si aquello que sucede en economías desarrolladas, sucede de igual manera en las economías emergentes de Latinoamérica.

A continuación se presenta y analiza la evolución de los reportes de sostenibilidad presentados ante la GRI entre 2010 y 2015 en las principales economías de Latinoamérica. Adicionalmente se presentan datos cuantitativos y cualitativos para entender el comportamiento de las prácticas de reporteo en el contexto latinoamericano.

\section{METODOLOGÍA}

El presente artículo fue desarrollado mediante un tipo de estudio mixto con un diseño explicativo secuencial (Hernández, Fernández y Baptista, 2014), y se estructura a partir de dos fases. La primera consistió en el análisis de los datos cuantitativos extraídos de los reportes solicitados vía correo electrónico a la oficina central de la GRI, con sede en Países Bajos, que suministró un archivo en formato Excel, el cual contenía la información desde 1996 hasta principios de 2016. Estos se ordenaron y tabularon de tal forma que se pudieran graficar y extraer del documento, y se seleccionaron los que adicionalmente cumplieran con los siguientes criterios: reportes de los países que, además de pertenecer a Latinoamérica durante el periodo analizado, presentaron entre todos ellos una sumatoria de más del $80 \%$ del PIB del total de los países que pertenecen a esta región (Banco Mundial, 2016).

Una vez seleccionada la información se procedió a analizar la evolución de los siguientes países: Argentina, Brasil, Chile, Colombia y México, los cuales ostentaron el 84.03\% del PIB de Latinoamérica desde 2010 hasta 2015. Adicionalmente se analizaron los cambios presentados y las variables por describir, datos que son comparaciones por año, por países, por sectores y por tamaño de empresas.

La segunda fase consistió en recabar datos a través de entrevistas semiestructuradas con previa firma del consentimiento informado de los entrevistados, los cuales se seleccionaron teniendo en cuenta que cumplieran algunos de los siguientes criterios: académicos expertos en RSE, responsables de elaborar informes de sostenibilidad que usaran la guía de la GRI e integrantes de las partes interesadas de las empresas que presentan este tipo de reportes. La formulación de las preguntas para los entrevistados se hizo teniendo en cuenta la clasificación de actividades de responsabilidad social propuesta por Carroll (1999). Las entrevistas fueron transcritas y 
codificadas de acuerdo con los lineamientos de análisis cualitativo sugeridos a partir de la metodología de investigación (Hernández et al., 2014).

- Perfiles de las personas entrevistadas:

E1: Estudiante de doctorado con artículos publicados en revistas indexadas sobre reporteo en RSE.

E2: Consultora en elaboración de informes de RSE con la metodología GRI.

E3: Responsable de presentar informe de RSE con la metodología GRI.

E4: Empleada de una empresa del sector de la salud que reporta ante la GRI.

E5: Directivo intermedio de empresa que reporta ante la GRI.

E6: Subdirector de sostenibilidad de empresa multinacional del sector cementos y concreto, encargado de coordinar la redacción del reporte que se presenta ante la GRI.

Finalmente, los datos cuantitativos y cualitativos fueron integrados con el objetivo de interpretar y explicar los descubrimientos de la fase inicial y asimismo profundizar en ellos.

En la tabla 1 se presentan las variables cualitativas y cuantitativas usadas para la recolección y el análisis de datos.

Tabla 1. Variables cuantitativas y cualitativas que se tuvieron en cuenta para el análisis de resultados

\begin{tabular}{|l|l|}
\hline \multicolumn{1}{|c|}{ Variables cuantitativas } & \multicolumn{1}{c|}{ Variables cualitativas } \\
\hline Número de reportes por año & Razones comerciales \\
\hline Número de reportes por países & Razones legales \\
\hline Número de reportes por sectores & Razones morales \\
\hline Número de reportes por tamaño & Razones filantrópicas \\
\hline
\end{tabular}

Fuente: elaboración propia.

\section{RESULTADOS Y DISCUSIÓN}

A continuación se presentan los hallazgos a través de estadística descriptiva y comparativa, teniendo en cuenta las variables planteadas y presentadas a través de tablas y gráficas, según sea el caso. Los resultados son analizados buscando explicar la evolución en la cantidad de informes presentados bajo la metodología GRI en las cinco economías más grandes de Latinoamérica.

\section{Economías más grandes de Latinoamérica}

En la tabla 2 se presenta el escalafón que hace anualmente el Banco Mundial, el cual determinó las economías más grandes de Latinoamérica en 2016.

Tabla 2. Listado de países de Latinoamérica por PIB en 2016

\begin{tabular}{|c|c|c|}
\hline Posición & País & PIB 2016 (USD) \\
\hline 1 & Brasil & USD 1.796.186.586.414 \\
\hline 2 & México & USD 1.045.998.068.645 \\
\hline 3 & Argentina & USD 545.866.164.478 \\
\hline 4 & Colombia & USD 282.462.548.889 \\
\hline 5 & Chile & USD 247.027.912.574 \\
\hline 6 & Perú & USD 192.093.512.185 \\
\hline 7 & Ecuador & USD 97.802.211.000 \\
\hline
\end{tabular}

Fuente: elaboración propia con base en Banco Mundial, 2016.

\section{Número de reportes de los países analizados}

En la figura 1, se muestra el número de reportes presentados ante el GRI por año y por país, haciendo un análisis comparativo entre el 2010 y el 2015.

Se observa que el país que más registros realizó fue Brasil con un total de 1326 informes, seguido por Colombia con 701. El país que menos reportes totalizó fue Chile con 294. También se puede observar 
que la mayor tasa de crecimiento fue la de Colombia con un $500 \%$ en el periodo de 2010 a 2015. La tasa de crecimiento total de los cinco países fue de $137.2 \%$, en la que se puede observar que en 2010 se reportaron 301 informes frente a 714 de 2015. Estos resultados concuerdan en gran parte con el artículo de Ortas y Moneva (2011), quienes analizaron la evolución de los reportes presentados desde 1999 hasta 2009. Encontraron que para el último año estudiado los países de Latinoamérica que más reportes presentaron fueron en su respectivo orden Brasil con 78 informes, Chile con 38 y México y Colombia con 19.

Figura 1. Número de reportes de los cinco países seleccionados entre 2010 y 2015

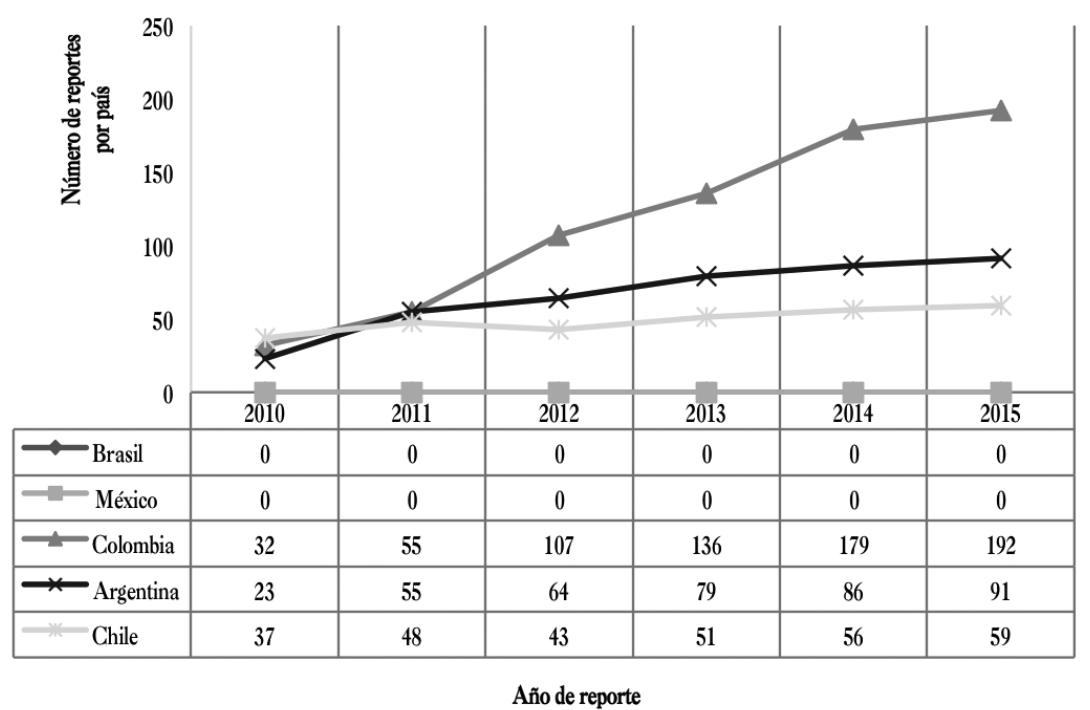

Fuente: elaboración propia con base en GRI, 2016.

\section{Número de reportes por tamaño de empresa}

En esta sección se presenta el número de reportes por tamaño de empresa según la siguiente clasificación: multinacionales, gran empresa y pequeña y mediana empresa (pyme).

\section{Análisis multinacionales}

A continuación, se presentan los datos recopilados de las empresas multinacionales. Se entiende por empresa multinacional aquella que tiene más de 250 empleados, negocios por más de 50 millones de euros, o un patrimonio mayor a 53 millones de euros, y operaciones en más de un país (GRI, 2017b).

En la gráfica se observa que entre 2012 y 2015 las empresas multinacionales de Colombia, Argentina y Brasil reportaron de forma constante y en similares proporciones desde 2011. Contrariamente, el menor porcentaje de reportes fue de Chile. En la sumatoria de los cinco países se evidenció un crecimiento sostenido en el número de reportes entre 2010 y 2013, pero se estabiliza desde 2013 hasta 2015. El crecimiento de todos los países es del $225 \%$ en los seis años de la muestra, pasando de 36 en 2010 a 117 en 2015. 
Fig̉ura 2. Evolución multinacionales 2010-2015

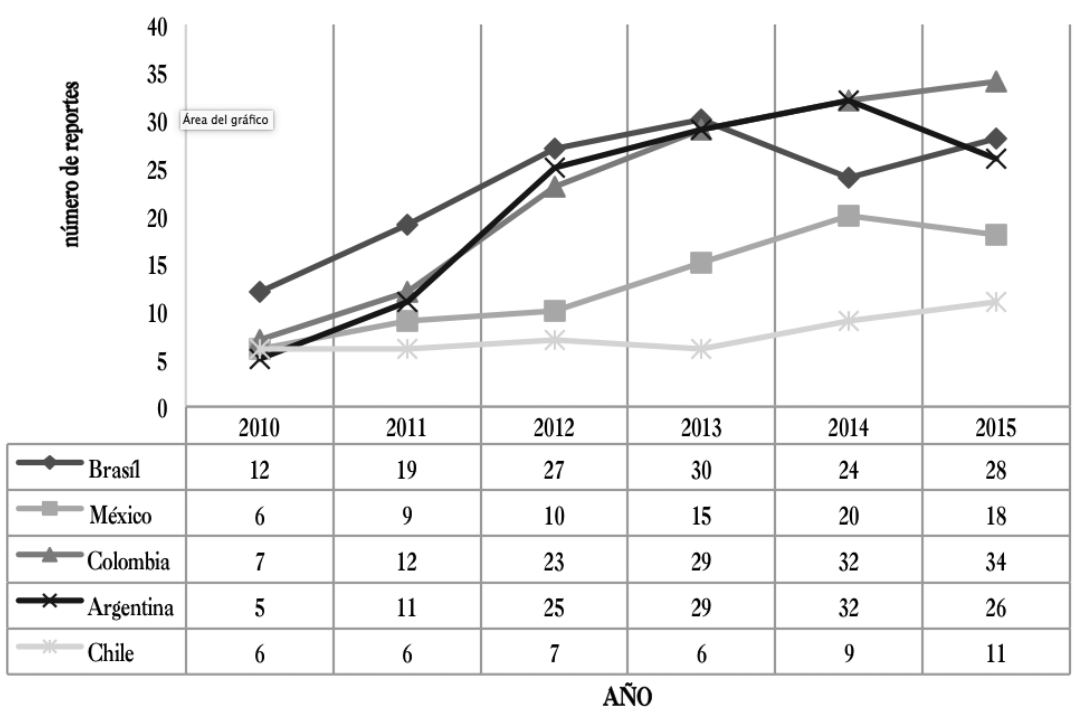

Fuente: elaboración propia con base en GRI, 2016.

Este comportamiento, según explican tres de los entrevistados, se debe a que tales reportes están principalmente pensados para empresas multinacionales que responden a grupos inversionistas o tienen clientes corporativos que los exigen para hacer negocios con ellos. Mencionan también que son empresas con operaciones en diferentes países, algunos de estos con regulación para presentar reportes si se cumplen ciertas condiciones.

De lo anterior se puede inferir que el comportamiento de las multinacionales puede obedecer a una presión de grupos inversores y del gobierno. Esto puede variar en función del país y el sector productivo. Asimismo, las multinacionales demuestran con los indicadores de crecimiento año a año su capacidad para desarrollar los informes según la región donde producen y su capacidad de adaptación a la guía correspondiente.

\section{Análisis gran empresa}

A continuación se presentan los resultados y el análisis de la gran empresa. Se define gran empresa como aquella que tiene más de 250 empleados y negocios por más de 50 millones de euros o un patrimonio igual o mayor a 53 millones de euros (GRI, 2017b).

Respecto a lo que se reporta sobre la gran empresa, se evidencia un crecimiento de este segmento en Brasil entre 2010 y 2013, pero un estancamiento entre 2013 y 2015. Por su parte, en Colombia se presentó un crecimiento sostenido durante los seis años, al igual que en Argentina. Con respecto a la gran empresa el país que más creció en los seis años fue Colombia con un 368.18\% y el que menos, Brasil con $39.33 \%$. El crecimiento de los cinco países fue del $110.17 \%$, pasando de 236 en 2010 a 496 en 2015. 
Figura 3. Evolución gran empresa 2010-2015

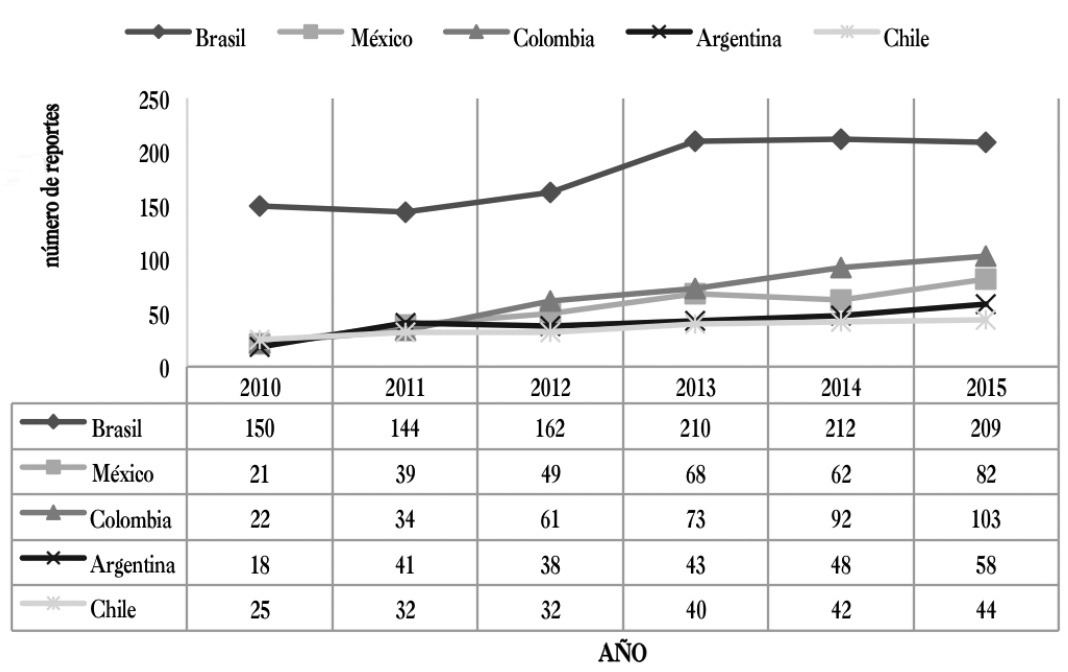

Fuente: elaboración propia con base en GRI, 2016.

De acuerdo con las entrevistas, estos fenómenos se explican a partir de la necesidad de las empresas de generar una buena reputación ya que necesitan verse bien ante los inversionistas, existentes y potenciales, presentes en la comunidad en donde operan diariamente. También está la cuestión del mejoramiento continuo ya que el sistema de GRI les permite reconocer en dónde se está actuando mal para poder cambiar el rumbo.

Por último, cuatro de los entrevistados mencionaron que las empresas reportaban por competitividad ya que muchos sectores tienen la costumbre de reportar, y las empresas que no lo hacen se ven afectadas y pueden perder negocios importantes o frenar su crecimiento.

\section{Análisis pyme}

A continuación se presentan los resultados y el análisis de las pymes. Estas se definen como las empresas que tienen menos de 250 empleados, operaciones por menos de 50 millones de euros o un patrimonio menor a 53 millones de euros.
En el segmento de las pymes se encontró que en 2010 México fue el que más reportes presentó, pero mantuvo un crecimiento muy bajo, si se observa consecutivamente hasta 2015. En contraste, Colombia, que en 2010 ocupaba la cuarta posición, fue el país que más reportó crecimiento constante ya que para 2015 generó 55 informes, seguido en ese mismo año de Brasil con 25. Se evidencia que México y Chile decrecieron en sus informes. El crecimiento de los cinco países fue del $236.6 \%$, pasando de 30 informes en 2010 a 92 en 2016.

Según los entrevistados, las pymes reportan en un nivel muy bajo porque el GRI no está adecuado a pequeñas empresas y estas no pueden cumplir con todos los requerimientos para un buen informe. Otro de los motivos por los cuales la cantidad de reportes tiende a ser baja es el costo en que deben incurrir las empresas. También se mencionó que las pymes que reportan lo hacen con el ánimo de crecer y hacer negocios con otras empresas de gran tamaño, que exigen demostrar un comportamiento responsable. 
Figura 4. Evolución pyme 2010-2015

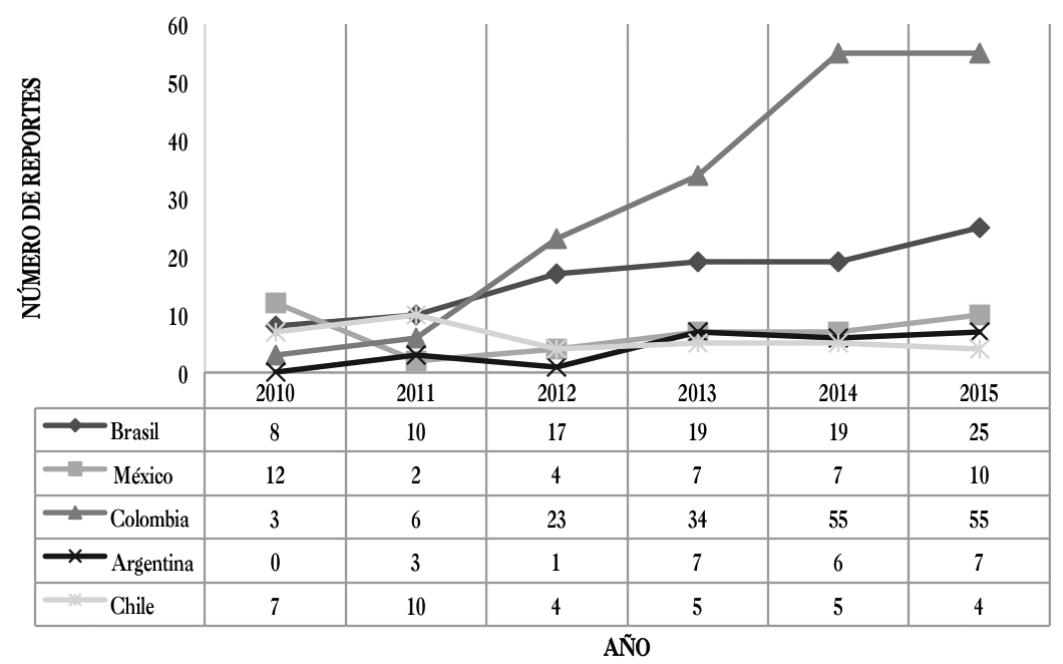

Fuente: elaboración propia con base en GRI, 2016.

\section{Sectores industriales que más reportaron}

En la figura 5 se presenta el número de reportes de los diez sectores que más reportaron entre 2010 y 2015.
Asimismo, en la tabla 3 se presenta el número de reportes por sector y por países.

Fiǵura 5. Sectores con mayor número de reportes entre 2010 y 1015 de las cinco economías latinoamericanas más grandes

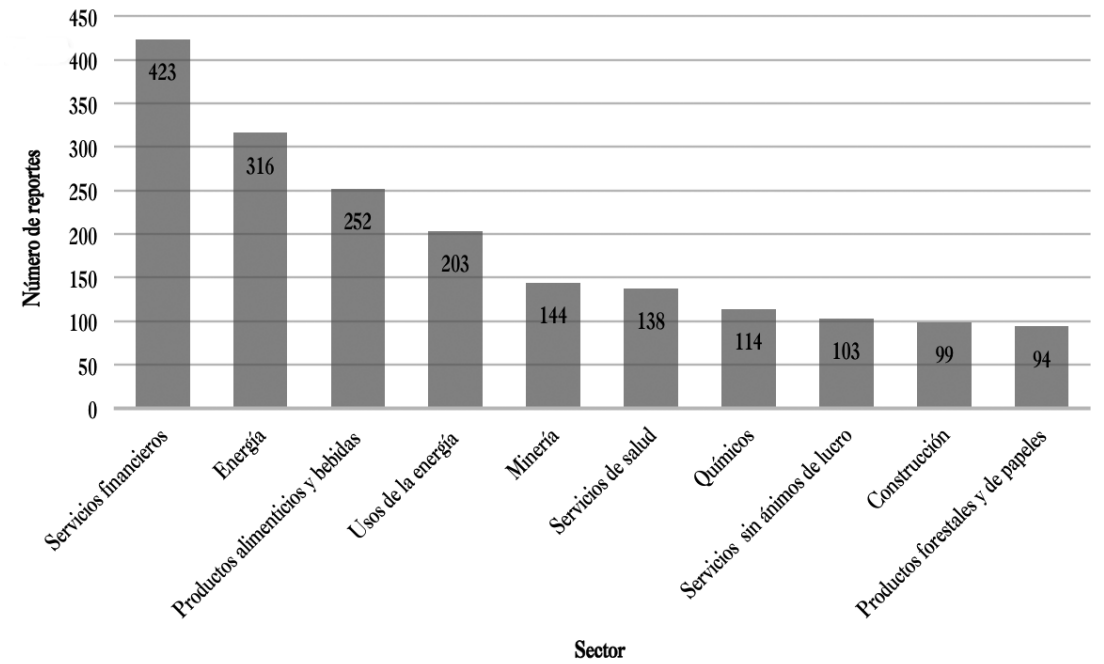

Fuente: elaboración propia con base en GRI, 2016. 
Tabla 3. Número de reportes desagregados por sector y país entre 2010 y 2015

\begin{tabular}{|l|c|c|c|c|c|}
\hline Sector & Brasil & Colombia & México & Argentina & Chile \\
\hline Servicios financieros & 147 & 103 & 79 & 66 & 28 \\
\hline Energía & 147 & 88 & 39 & 18 & 24 \\
\hline Alimentos y bebidas & 63 & 43 & 74 & 41 & 31 \\
\hline Usos de la energía & 100 & 57 & 19 & 24 & 3 \\
\hline Minería & 28 & 22 & 20 & 17 & 57 \\
\hline Servicios de salud & 97 & 16 & 16 & 8 & 5 \\
\hline Químicos & 50 & 10 & 31 & 12 & 11 \\
\hline $\begin{array}{l}\text { Servicios sin ánimo } \\
\text { de lucro }\end{array}$ & 20 & 57 & 17 & 4 & 5 \\
\hline Construcción & 47 & 17 & 25 & 5 & 5 \\
\hline $\begin{array}{l}\text { Productos forestales } \\
\text { y de papel }\end{array}$ & 59 & 7 & 15 & 1 & 12 \\
\hline
\end{tabular}

Fuente: elaboración propia con base en GRI, 2016.

En la figura 5 y la tabla 3 se encuentra que las empresas del sector de servicios financieros son las que más reportan en todos los países, con excepción de Chile, en donde son más frecuentes los reportes en minería. En el sector de productos forestales y de papel, Brasil aventaja por mucho a los otros cuatro países analizados. Después del sector financiero, los sectores de energía y usos de la energía reportan más frecuentemente en todos los países de la muestra.

Gómez y Quintanilla (2012) evidencian que los sectores que más reportan en Latinoamérica son energía, usos de la energía, empresas financieras, minería, agricultura y alimentos, en ese orden, lo que coincide con la información de los países del presente documento.

En el sector financiero Brasil es el único que está por encima de la media todos los años; Chile, por su parte, está por debajo todos los años. Colombia es el país con mayor crecimiento, reportando una variación del $480 \%$, seguido de México y Chile con un $200 \%$ en el periodo analizado. Chile es el país que presenta menos reportes de empresas financieras entre las cinco economías, con
28 informes entregados. En la región, los países que más reportan son Brasil con 147 informes, seguido por Colombia con 88. En cuanto al crecimiento del sector, entre 2011 y 2013 creció constantemente; pero en los dos últimos periodos redujo su ritmo a crecimientos de un solo dígito (GRI, 2016).

En el sector energía Brasil está por encima de la media todos los años de la muestra; Colombia lo está en los últimos cuatro años y los demás países siempre están por debajo. Colombia fue el país que más creció en el número de reportes en todos los años analizados con un $525 \%$, seguido por Argentina con un 500\%. El crecimiento del sector ha sido constante y en total creció un $53 \%$, pasando de 34 informes presentados en 2010 a 72 en 2015 (GRI, 2016).

En el sector eléctrico Talbot y Boiral (2018) encontraron que las empresas en su mayoría presentan informes con deficiencias y que hay un número creciente de estas. La magnitud de los impactos ambientales del sector y la necesidad de cuidar la reputación podrían ser causas de este fenómeno.

En el sector de alimentos y bebidas Brasil y México están por encima de la media todos los años de la muestra y Argentina y Colombia están siempre por debajo. El país que más creció en los cinco años fue Argentina con $400 \%$, pasando de 2 reportes en 2010 a 10 en 2015 . La presentación de informes en el sector ha crecido todos los años. Sin embargo, en 2014 solamente se incrementó en un $6 \%$ debido a que Brasil y México hicieron la misma cantidad de reportes que en 2013 y Chile reportó uno menos (GRI, 2016).

En el sector de utilidades de la energía Brasil disminuyó sus reportes entre 2010 y 2015 presentando un decrecimiento del 42\%, Colombia presentó un crecimiento durante todos los años, México no reportó en ningún año y Chile solo lo hizo en 2010, 2011 y 2013 una vez por 
año. El país que más creció durante los cinco años fue Argentina con un $75 \%$, seguido por Colombia. El sector creció en 2012 y 2013, pero decreció entre 2014 y 2015, y en 2014 no creció con respecto a 2013 (GRI, 2016).

En el sector minería Chile estuvo por encima de la media todos los años analizados y es el país que más reportó en todos los periodos con un total de 57 reportes. Brasil estuvo por encima de la media de 2012 a 2014, pero en 2015 volvió a quedar por debajo. La media del sector creció $41 \%$ durante los cinco años, y el incremento del sector en los dos primeros fue del $24 \%$, pero disminuyó en 2013, 2014 y 2015 en un 14\%. Al respecto Boiral y Henri (2017) afirman que hay una falta de estandarización en los informes de este sector, que puede o no extenderse a otros sectores. Eso hace que los informes no sean comparables entre sí, y por este motivo no se puede determinar si son o no de alta calidad.

\section{Análisis del ejercicio de reporteo a la luz de las cuatro clases de responsabilidades sociales identificadas por Carroll}

A partir del análisis de los resultados cuantitativos se encuentra que en general todos los países vienen creciendo. En el total es Brasil el país que más informes presentó, seguido de Colombia y México. Consecuentemente, el país que más creció es Colombia ya que presentó un crecimiento constante durante los cinco años. Por su parte, Brasil creció hasta 2013, pero en los dos últimos años su crecimiento se detuvo. Por último, México, Argentina y Chile crecieron en menor medida.

En el segmento de las multinacionales Colombia es el país que más informes presentó, seguido de Brasil y México. En cuanto a la gran empresa, Brasil es el país que aventaja ampliamente a los otros cuatro; sin embargo, en los últimos dos años dejó de crecer, a diferencia de los cuatro países restantes, los cuales mantuvieron un crecimiento constante durante los cinco años. En cuanto a las pymes, Colombia fue el país que más resultados generó, a excepción de Brasil, que lo dobló en resultados. Sin embargo, se reporta muy poco en todos los países en esta categoría, teniendo en cuenta que en este segmento está el mayor número de empresas en todos los países analizados.

Para explicar este fenómeno de crecimiento se llevó a cabo un proceso de indagación con expertos. Las preguntas a estos se desprenden de la propuesta de Carroll (1999). En ella la RSE se puede entender en cuatro niveles, a saber: económico, legal, ético y filantrópico. A continuación, el análisis de los hallazgos del proceso de indagación.

\section{Nivel económico}

Los entrevistados encuentran que las empresas reportan para legitimar sus actos dentro de la comunidad en la cual están actuando y de esta forma generar visibilidad frente a sus competidores y posibles inversores. También con el fin de ahorrar al identificar costos ocultos. Por ejemplo, aquellos generados por multas, demandas o futuras regulaciones. De esta forma, la comunidad dará validez a sus operaciones al percibir por parte de la empresa una preocupación por el bienestar de las partes interesadas.

Talbot y Boiral (2018) explican que las empresas muchas veces adelantan acciones correctivas, y no preventivas, destinadas a proteger su imagen corporativa. Estas conclusiones concuerdan con las de Guix, Bonilla-Priego y Font (2017), quienes mencionan que la presentación de informes de sostenibilidad es más un ejercicio de legitimación que de rendición de cuentas, y que el enfoque de identificación de partes interesadas no demuestra la transparencia de la organización.

Perez-Batres, Miller y Pisani (2010) señalan que las empresas latinoamericanas que tienen vínculos con empresas europeas tienen mayores posibilidades de reportar en 
comparación con las que tienen vínculos con empresas estadounidenses. Esto demuestra que ellas reportan, en gran medida, por cuestiones económicas al buscar mantener los estándares que les exigen sus casas matrices.

\section{Nivel legal}

Las personas entrevistadas aseguran que el interés de las empresas es antes que nada ajustarse a las normas de cada país. Si de alguna manera las practicas exigidas en un país particular se pueden aplicar en otras sucursales o en otros territorios en donde se tengan operaciones, no tienen problema en implementarlas como parte de sus actividades en RSE. También mencionan que en los casos en los que se quiera acceder a algún tipo de acreditación de calidad por parte del Gobierno, es necesaria la presentación de informes de sostenibilidad. Al respecto, Thijssens et al. (2016) ponen en evidencia que algunos de los informes de sostenibilidad, particularmente de las compañías químicas y petroleras, pueden estar motivados por requisitos legales.

\section{Nivel ético}

Guix et al. (2017) afirman que, para que el diálogo sea efectivo, es necesario que exista evidencia del impacto que la participación de las partes interesadas ha tenido en el comportamiento de la organización. La mayoría de los informes de sostenibilidad analizados no incluyen detalles de la retroalimentación recibida de las partes interesadas; tampoco aclaran en qué medida dicha retroalimentación influye en el proceso de toma de decisiones de la organización. En este punto, las personas encuentran que las empresas reportan por convicción pues realmente les interesa que la comunidad en la que actúan se sienta bien y cree sentido de pertenencia hacia estas.

Manetti (2011) afirma que uno de los factores más importantes en la redacción de este tipo de documentos es la relación que las empresas forjaron con las partes interesadas. Sin embargo, encuentra que la participación de estas no se evidencia en los resultados finales, y aunque no lo puede afirmar, tiene la hipótesis de que toda empresa adopta un tipo de lenguaje decididamente retórico, carente de indicaciones concretas, sobre las externalidades negativas producidas, en un intento por alcanzar sus objetivos de autolegitimación, por lo que las partes interesadas no son realmente tomadas en cuenta.

\section{Nivel filantrópico}

En este nivel a las empresas verdaderamente les preocupa la comunidad, se sienten parte de ella y esta les corresponde de igual forma; siempre están buscando que las partes interesadas en efecto mejoren su calidad de vida. Algunos de los entrevistados realmente creen que la empresa solo puede crecer de la mano de la comunidad y no de otra forma; que debe estar en contacto permanente con la comunidad y atendiendo de una forma sincera y transparente todos sus requerimientos haciéndole saber que es una empresa y debe generar ganancias, pero que no por esto afectará a la comunidad. Thijssens et al. (2016) mencionan algunos factores internos que facilitan la redacción de este tipo de informes, los cuales se relacionan con estilos y actitudes de los altos directivos, como la influencia del número de directores externos no empresariales, la presencia de un comité de sostenibilidad y cambios en el presidente de la compañía. Asimismo Guix et al. (2017) argumentan que no solo importa el nivel de responsabilidad, sino también la alineación de la estrategia con las expectativas de las partes interesadas.

\section{CONCLUSIONES}

La presentación de reportes de sostenibilidad viene creciendo de una manera importante en todos los países analizados, aunque Brasil particularmente presenta un 
estancamiento. Por su parte, Colombia exhibe el mayor crecimiento en particular en los sectores financiero y energético. A partir de los datos expuestos previamente se hace evidente que para América Latina la economía más fuerte es la de Brasil. Esto se confirma con los datos publicados por el Banco Mundial, en los cuales se evidencia que es la séptima economía más grande a nivel global. Posiblemente esta situación unida a las políticas regulatorias del gobierno brasileño, el cual busca implementar la generación de reportes en RSE, explique que sea el que más reporta de los países analizados (Conceição, Dourado y Silva, 2012; Alonso-Almeida, Marimon y Llach, 2015; Sierra-García, García-Benau, y Zorio, 2014).

Colombia es la segunda nación con el mayor número de reportes entre los países analizados y la de mayor crecimiento constante. Esto podría explicarse por un aumento en el número de reportes de las pymes en comparación con la gran empresa y las multinacionales. El crecimiento del reporteo de las pymes en Colombia es atípico en Latinoamérica y debe ser un asunto de interés en futuras investigaciones. En cuanto al tamaño y la antigüedad se encontró que las empresas a medida que son más grandes y más antiguas desarrollan más actividades de RSE. No obstante, la asignación de presupuesto solo se ve reflejada en las empresas de mayor tamaño (Yépez et al., 2015). Este fenómeno se puede explicar por la disponibilidad de recursos de las empresas, que permite contratar a consultores renombrados, los cuales saben comunicar el resultado de sus acciones adecuadamente para que esto no perjudique su imagen corporativa (De Beelde y Tuybens, 2015; Conceição et al., 2012).

Otro factor que incide en el constante crecimiento de los reportes es la presión que ejercen los mercados y las organizaciones internacionales en favor de la sostenibilidad (Griesse, 2007). La gran empresa tiene un papel fundamental frente a la toma de decisiones que pueden influir directamente en temas de sostenibilidad en la estructura de una nación (Vicente, Vaz y De Noronha, 2015; Eric Vaz, y Teresa de Noronha, 2015). Según Legna (2007) reportar ante la GRI representa una herramienta que hace visibles los resultados económicos, sociales y ambientales para los distintos grupos de interés. Permite además evidenciar una mejora en la imagen corporativa y brindar seguridad, satisfacción y transparencia a los inversores (De Beelde y Tuybens, 2015).

Uno de los aspectos que justifica la implementación de reportes GRI en el sector financiero obedece a que son empresas estratégicas ya que generan un importante impacto en el funcionamiento y progreso de la economía, y por ende, en el progreso social de un país (Bravino, Margaria y Heredia, 2016). Es por esto que países como Argentina (Instituto Argentino de Responsabilidad Social Empresarial), Brasil (Índice de Sostenibilidad Empresarial), Colombia (Fondo Inversor de Bolsa de Valores de Colombia) y México (índice IPC de la Bolsa Mexicana de Valores) cuentan con entidades de alto nivel comprometidas con el desarrollo sostenible. También es una forma de reforzar su imagen corporativa ante eventualidades como el caso de la crisis financiera de 2008 (Alonso-Almeida et al., 2015), tras la cual se dieron lineamientos nuevos y diferentes, de modo que el sector logró construir y mantener su legitimidad y licencia social (Rabasedas, Barco, Sarrúa y Alberto, 2016). No obstante, Gómez y Quintanilla (2012) critican estos informes de sostenibilidad mencionando que mientras todos los diagnósticos muestran la irresponsabilidad del sector financiero, en aquellos las instituciones que los producen se califican a sí mismas como muy apegadas a la RSE con la anuencia de sus auditores.

Por otro lado, Haro, Saraite, Caba y Gálvez (2016) mencionan que en relación con las comunidades y el medio ambiente el sector energético siempre se ha visto como una fuente de impacto negativo debido a que sus áreas 
de trabajo se ubican en zonas rurales con poblaciones pobres y producen daños en fuentes hídricas y bosques tropicales, lo que afecta la flora y fauna de las regiones. Alonso-Almeida et al. (2015) mencionan que por este motivo se reporta: para limpiar la imagen debido a las polémicas que se están generando en países de Latinoamérica frente a la reserva y explotación de dichos recursos y del medio ambiente.

Por su parte, al analizar el sector minero se identifica que es representativo para Chile. Los reportes tratan de mostrar la cara amable de la empresa, y por eso se encuentra que no publican toda la información debido a los conflictos de interés entre las empresas y las partes interesadas (Murguía y Böhling, 2013).

Se puede pensar que reportar resulta ser una estrategia efectiva para mejorar o mantener la imagen de las organizaciones frente a sus partes interesadas, cumplir con regulaciones en los países en los que se está operando, prevenir futuras regulaciones y mejorar la competitividad. No obstante, dada la necesidad de incrementar sus rendimientos financieros y teniendo en cuenta que este tipo de informes es estrictamente voluntario, los resultados que se muestran a la comunidad son parciales. En este sentido, Calixto (2013) encuentra que las compañías de Latinoamérica no ofrecen información socioambiental en sus informes anuales o en informes específicos sobre el tema.

Landrum y Ohsowski (2018) sostienen que la gran mayoría de las empresas reporta principalmente para lograr mejores beneficios económicos y no con el objetivo de construir relaciones duraderas con las partes interesadas. Las empresas que deben cuidar su reputación están más presionadas por los grupos de interés en su comportamiento ético, así como por el mercado y las empresas internacionales. Esto probablemente se explica debido a que muchas economías emergentes de todo el mundo adoptaron el modelo anglosajón de gobierno corporativo y es evidente la eficacia de dichos mecanismos, entre los que se incluye la cultura del reporteo social (Nguyen y Boubaker, 2014).

Las empresas multinacionales reportan porque en algunos de los países en donde tienen operaciones existen normas regulatorias para este tipo de actividades 0 existen claras tendencias para regular en este sentido. También lo hacen para estandarizar sus compañías a nivel global. Las empresas nacionales de gran tamaño reportan por competitividad ya que sienten que pueden perder terreno frente a sus rivales, dejar de percibir contratos con empresas multinacionales o frenar su curva de crecimiento si no lo hacen.

En las pymes se presenta un crecimiento constante de los reportes; sin embargo, la cantidad es baja en todos los países. Esto podría deberse a motivos financieros. Se estima que empresas de este tamaño asumen tal tipo de iniciativas con el objetivo de lograr hacer negocios con compañías más grandes. Las empresas a nivel regional están tomando conciencia acerca de la RSE y su impacto dentro de la sociedad ya que es evidente que aportar un valor agregado a las partes interesadas les representa beneficios importantes como perdurar en el tiempo. Sin embargo, este tipo de gestiones representan un alto costo. Por lo tanto, los gobiernos deberían crear iniciativas que incluyan a las pymes en temas de sostenibilidad.

Aunque claramente este tipo de informes es de carácter voluntario, la tendencia creciente es cada vez más notoria dentro de las cinco naciones analizadas. El afán de las empresas por permanecer a la cabeza dentro de sus respectivos sectores y al entrar en mercados de Europa y Norteamérica hace que este tipo de iniciativas se tome cada vez más en serio. Las empresas multinacionales y las de gran tamaño parecen haberlo entendido así, y las pymes están empezando a tomar decisiones en este sentido. 
Las personas entrevistadas vienen estudiando el reporteo ante la GRI, bien sea académicamente o como parte de una empresa que genera estos informes, y coinciden en que es un reporte que debe estar dirigido a las partes interesadas. Sin embargo, reconocen que son muy largos y complicados en su interpretación, y que los esfuerzos que hacen para socializarlos son insuficientes o simplemente no cumplen su objetivo. Esto indica que las empresas deben prestar atención especial a la divulgación de sus acciones, cuestionándose sobre la manera más efectiva para divulgar su impacto de acuerdo con el perfil del grupo de interés.

Finalmente, se observa que el aumento de la tendencia en la generación de este tipo de informes, los cuales representan una inversión de múltiples recursos, se ve recompensado con el mejoramiento de los procesos internos de las empresas, el aumento de su competitividad, la mejora de imagen frente a posibles inversionistas y la construcción de una relación armónica con su entorno.

\section{REFERENCIAS}

Alonso-Almeida, M. del M., Marimon, F. y Llach, J. (2015). Difusión de las memorias de sostenibilidad en Latinoamérica: análisis territorial y sectorial. Estudios Gerenciales, 31(135), 139-149. Doi: https://doi. org/10.1016/j.estger.2015.01.002

Archel, P. y Husillos, J. (2009). Divulgación de información social y medioambiental: una revisión de la literatura. En W. Rojas (ed.), Irrupciones significativas para pensar la contabilidad (pp. 13-60). Cali: Universidad del Valle.

Baker, C. R. (2005). What is the meaning of "the public interest"?: Examining the ideology of the American public accounting profession. Accounting, Auditing $\mathbb{C}^{\circ}$ Accountability Journal, 18(5), 690-703. Doi: https:// doi.org/10.1108/09513570510620510

Banco Mundial (2016). Datos. PIB (US\$ a precios actuales). Recuperado de https://tinyurl.com/y3tbpub4

Boiral, O. y Henri, J. F. (2017). Is sustainability performance comparable? A study of GRI Reports of mining organizations. Business and Society, 56(2), 283317. Doi: https://doi.org/10.1177/0007650315576134

Bravino, L. S., Margaria, O. A. y Heredia, E. R. (2016). Los servicios financieros y la sustentabilidad. Pistas Educativas, 38(123), 120-137.

Brown, H. S., De Jong, M. y Levy, D. L. (2009). Building institutions based on information disclosure: Lessons from GRI's sustainability reporting. Journal of Cleaner Production, 17, 571-580. Doi: https:// doi.org/10.1016/j.jclepro.2008.12.009

Calixto, L. (2013). A divulgação de relatórios de sustentabilidade na América Latina: um estudo comparativo. Revista de Administração (São Paulo), 48(4), 828-842.

Carroll, A. B. (1999). Corporate social responsibility: Evolution of a definitional construct. Business and Society, 38(3), 268-295. Doi: https://doi. org/10.1177/000765039903800303

Chapple, W. y Moon, J. (2005). Corporate Social Responsibility (CSR) in Asia: A seven-country study of CSR web site reporting. Business \&̊ Society, 44(4), 415-441. Doi: https://doi.org/10.1177/0007650305281658

Conceição, S., Dourado, G. y Silva, S. (2012). Global Reporting Initiative (GRI) - um estudo exploratório da prática de evidenciação em sustentabilidade 
empresarial na América Latina. Revista de Gestão, Finanças e Contabilidade, 2(3), 17-38.

Correa, M. E., Flynn, S. y Amit, A. (2004). Responsabilidad social corporativa en América Latina: una visión empresarial. Serie Medio Ambiente y Desarrollo, 85. Recuperado de https://tinyurl.com/y6auyoy9

De Beelde, I. y Tuybens, S. (2015). Enhancing the credibility of reporting on corporate social responsibility in Europe. Business Strategy and the Environment, 24(3), 190-216. Doi: https://doi.org/10.1002/ bse. 1814

De la Cuesta, M. (2004). El porqué de la responsabilidad social corporativa. Boletín Económico de ICE, Información Comercial Española, 2813, 45-58.

Donaldson, T. y Preston, L. E. (1995). The stakeholder theory of the corporation: Concepts, evidence and implications. Academy of Management Revierw, 23(1), 65-91.

Duque, Y. V., Cardona, M. y Rendón, J. A. (2013). Responsabilidad social empresarial: teorías, índices, estándares y certificaciones. Cuadernos de Administración, 29(50), 196-206. Doi: https://doi. org/10.25100/cdea.v29i50.55

English, D. M. y Schooley, D. K. (2014). The evolution of sustainability reporting. CPA Journal, 84(3), 26-35.

Fasan, M. y Mio, C. (2017). Fostering stakeholder engagement: The role of materiality disclosure in integrated reporting. Business Strategy \& the Environment, 26(3), 288-305. Doi: https://doi.org/10.1002/ bse. 1917
García, R. F. (2009). Responsabilidad social corporativa. Una estrategia para conseguir imagen y reputación. Icono, 14(13), 95-124.

Gómez, M. y Quintanilla, D. A. (2012). Los informes de responsabilidad social empresarial: su evolución y tendencias en el contexto internacional y colombiano. Cuadernos de Contabilidad, 13(32), 121-158.

GRI (Global Reporting Initiative) (2015). Guía para la elaboración de memorias de sostenibilidad G4.

GRI (Global Reporting Initiative) (2016). GRI-Reports list complete 1999-2016 [Base de datos en Office Excel]. Ámsterdam: GRI.

GRI (Global Reporting Initiative) (2017a). About GRI. Recuperado de https://tinyurl.com/yyfvveqr.

GRI (Global Reporting Initiative) (2017b). Sustainability disclosure database. Data legend. Recuperado de https://tinyurl.com/y5crgj5t

Griesse, M. A. (2007). The geographic, political, and economic context for corporate social responsibility in Brazil. Journal of Business Ethics, 73(1), 21-37. Doi: https://doi.org/10.1007/s10551-006-9194-2

Guix, M., Bonilla-Priego, M. J. y Font, X. (2017). The process of sustainability reporting in international hotel groups: An analysis of stakeholder inclusiveness, materiality and responsiveness. Journal of Sustainable Tourism, 26(7), 1-22.

Haro, A., Saraite, L., Caba, C. y Gálvez, M. del M. (2016). Las empresas latinoamericanas del sector del petróleo y gas ante la información sobre sostenibilidad. TEC Empresarial, 10(1), 39-49. 
Hernández, R., Fernández, C. y Baptista, P. (2014). Metodologia de la investigación. México: McGraw-Hill Education.

Khan, M., Serafeim, G. y Yoon, A. (2016). Corporate sustainability: First evidence on materiality. The Accounting Review, 91(6), 1697-1724. Doi: https:// doi.org/10.2308/accr-51383

Kolk, A. (2003). Trends in sustainability reporting by the Fortune Global 250. Business Strategy \& the Environment, 12(5), 279-291. Doi: https://doi.org/10.1002/bse.37

Kolk, A. (2008). Sustainability, accountability and corporate governance: Exploring multinationals' reporting practices. Business Strategy \& the Environment, 17(1), 1-15. Doi: https://doi.org/10.1002/ bse. 511

Landrum, N. E. y Ohsowski, B. (2018). Identifying worldviews on corporate sustainability: A content analysis of corporate sustainability reports. Business Strategy \& the Environment, 27(1), 128-151. Doi: https://doi.org/10.1002/bse.1989

Legna, P. (2007). Reportes de sostenibilidad. Parte 1: concepto, beneficios y contenido. UAIS sustentabilidad. Recuperado de http://www.sustentabilidad. uai.edu.ar/pdf/rse/UAIS-RSE-300-001\%20-\%20 Reportes\%201.pdf

Lozano, J. F., Raufflet, E., Barrera, E. y García, C. (2012). Responsabilidad social empresarial. México: Pearson.

Manetti, G. (2011). The quality of stakeholder engagement in sustainability reporting: empirical evidence and critical points. Corporate Social Responsibility \& Environmental Management, 18(2), 110-122. Doi: https://doi.org/10.1002/csr.255
Matten, D. y Moon, J. (2008). "Implicit" and "explicit" CSR: A conceptual framework for a comparative understanding of corporate social responsibility. Academy of Management Revieres, 33(2), 404-424. Doi: https://doi.org/10.5465/amr.2008.31193458

McWilliams, A. y Siegel, D. (2001). Corporate social responsibility: A theory of the firm perspective. Academy of Management Reviere, 26(1), 117-127. Doi: https://doi.org/10.5465/amr.2001.4011987

Montoya, B. y Martínez, P. (2012). Responsabilidad social empresarial: una respuesta ética ante los desafíos globales. México: Fundacion Konrad Adenauer.

Moon, J. (2004). Government as a driver of corporate social responsibility: The UK in comparative perspective. International Centre for Corporate Social Responsibility, 20, 11-27.

Murguí, D. I. y Böhling, K. (2013). Sustainability reporting on large-scale mining conflicts: The case of Bajo de la Alumbrera, Argentina. Journal of Cleaner Production, 41, 202-209. Doi: https://doi. org/10.1016/j.jclepro.2012.10.012

Nguyen, D. K. y Boubaker, S. (2014). Corporate governance and corporate social responsibility: Emerging markets focus. Singapur: World Scientific.

Ortas, E. y Moneva, J. M. (2011). Origins and development of sustainability reporting: Analysis of the Latin American context. GCG: Revista de Globalización, Competitividady Gobernabilidad, 5(2), 16-37.

Perez-Batres, L. A., Miller, V. V. y Pisani, M. J. (2010). CSR, sustainability and the meaning of global reporting for Latin American corporations. Journal of Business Ethics, 91(suppl. 2). Doi: https://doi. org/10.1007/s10551-010-0614-y 
Porter, M. E. y Kramer, M. R. (2006). Strategy \& society: The link between competitive advantage and corporate social responsibility. Harvard Business Review, 84(12), 78-92.

Rabasedas, M. L., Barco, M. de los Á., Sarrúa, J. y Alberto, L. (2016). Análisis intersectorial de las memorias de sostenibilidad publicadas por empresas cotizadas en Argentina. SaberEs, 8(2), 133-160.

RobecoSAM y GRI (Global Reporting Initiative) (2015). Definición de la materialidad: lo que preparadores e inversores tienen en cuenta. Recuperado de https:// tinyurl.com/y4kjnyzs

S. Dhaliwal, D., Zhen Li, O., Tsang, A. y George Yang, Y. (2011). Voluntary nonfinancial disclosure and the cost of equity capital: The initiation of Corporate Social Responsibility Reporting. The Accounting Reviewe, 86(1), 59-100. Doi: https://doi.org/10.2308/ accr.00000005

Sierra-García, L., García-Benau, M. A. y Zorio, A. (2014). Credibilidad en latinoamérica del informe de responsabilidad social corporativa. Revista de Administração de Empresas, 54(1), 28-38.
Talbot, D. y Boiral, O. (2018). GHG reporting and impression management: An assessment of sustainability reports from the energy sector. Journal of Business Ethics, 147(2), 367-383. Doi: https://doi. org/10.1007/s10551-015-2979-4

Thijssens, T., Bollen, L. y Hassink, H. (2016). Managing sustainability reporting: Many ways to publish exemplary reports. Journal of Cleaner Production, 136(Part A), 86-101. Doi: https://doi.org/10.1016/j. jclepro.2016.01.098

Vicente, P., Vaz, E. y De Noronha, T. (2015). How corporations deal with reporting sustainability: Assessment using the multicriteria logistic biplot approach. Systems, 3(1), 6-26. Doi: https://doi. org/10.3390/systems3010006

Yépez, G. A., Camacho, M. T., Hernández, L. M., Cubillos, N. M., Franco, L., Rodríguez, M. A. y Cruz, J. (2015). Estado de la RSE en Colombia. Serie Avances de Entorno de los Negocios, 20.

Zorio, A., García-Benau, M. A. y Sierra, L. (2015). Aseguramiento del informe de sostenibilidad en España y Latinoamérica. Revista Innovar, 25(1), 85-102. Doi: https://doi.org/10.15446/innovar.v25n1Spe.53361 